tion $(E C 50=44.5 n M)$ that was reversed by Dipyridamole $(I C 50=0.3 \mu M)$. When Pannexin-1 and Connexin-43 were absent, Tenofovir did not increase OC number. Tenofovir increases Cathepsin K and NFATc1 mRNA levels during OC differentiation, and the effect was reverted by Dipyridamole. Tenofovir reduced bone formation in vivo $(19 \pm 2 \mu \mathrm{m}$ bone aposition vs $35 \pm 4 \mu \mathrm{m}$ untreated $p<0.05)$ and this effect was reverted in the presence of Dipyridamole $(30 \pm 3 \mu \mathrm{m}, \mathrm{p}<0.05$ vs Tenofovir alone). microCT revealed decrease BMD and altered trabecular bone in Tenofovir-treated mice, been reverted in the presence of Dipyridamole. TRAP-staining showed increased OC in vivo in Tenofovir-treated mice $(21 \pm 1$ vs $16 \pm 1 \mathrm{OC} / \mathrm{hpf}$ in untreated, $\mathrm{p}<0.005)$ that was reverted with Dipyridamole. Similar results were obtained for Cathepsin $\mathrm{K}$.

Conclusions: These results indicate that Tenofovir enhances OC differentiation and inhibits osteoblast differentiation by an adenosine-dependent mechanism and suggests that treatment with agents that increase local adenosine concentrations, like Dipyridamole, might prevent bone loss following Tenofovir treatment.

Disclosure of Interest: F. Conesa: None declared, P. Llamas: None declared, T. Wilder: None declared, P. Atencio: None declared, A. Cabello: None declared, M. Górgolas: None declared, B. Cronstein: None declared, R. Largo: None declared, G. Herrero-Beaumont: None declared, A. Mediero Grant/research support from: Instituto de Salud Carlos III, Fondos FEDER

DOI: 10.1136/annrheumdis-2017-eular.3207

\section{OP0187 TRANSGENIC DISRUPTION OF GLUCOCORTICOID-SIGNALING IN MATURE OSTEOBLASTS AND OSTEOCYTES ATTENUATES STRUCTURAL BONE DAMAGE IN A LONG-TERM MURINE K/BXN SERUM-INDUCED ARTHRITIS MODEL}

E. Wiebe ${ }^{1,2}$, C. Spies ${ }^{1,2}$, J. Tu ${ }^{2}$, T. Maleitzke ${ }^{1,2}$, Y. Zhang ${ }^{2}$, M. Seibel ${ }^{2,3}$, H. Zhou ${ }^{2}$, F. Buttgereit ${ }^{4} .{ }^{1}$ Department of Rheumatology and Clinical Immunology, Charité University Hospital, Berlin, Germany; ${ }^{2}$ Bone Research Program, ANZAC Research Institute, University of Sydney; ${ }^{3}$ Department of Endocrinology and Metabolism, Concord Repatriation Hospital, University of Sydney, Sydney, New South Wales, Australia; ${ }^{4}$ Department of Rheumatology and Clinical Immunology, Charité Universitätsmedizin Berlin, Berlin, Germany

Background: The role of endogenous glucocorticoids (GC) in bone metabolism in chronic inflammatory arthritis remains unclear. We have previously shown that disruption of GC-signaling in osteoblasts results in a marked attenuation of arthritis in the $\mathrm{K} / \mathrm{BxN}$ serum-induced and CAIA mouse model with preservation of bone volume and structure ${ }^{1,2}$

Objectives: In order to investigate the impact of endogenous GCs on bone erosion and turnover in chronic inflammatory arthritis, we now studied the effects of disrupted osteoblastic GC-signaling in a long-term murine arthritis model.

Methods: Intracellular GC-signaling in osteoblasts was disrupted by transgenic overexpression of 11 beta-hydroxysteroid dehydrogenase type 2 (11ß-HSD type 2) under the control of a type 1 collagen promoter. Arthritis was induced in 5-week old male transgenic (tg) mice and their wild-type (WT) littermates. In order to maintain a chronically active arthritis, mice were boosted on day 14 and 28 by subcutaneous injection of K/BxN serum, controls (CTR) received PBS, respectively. Severity of arthritis was assessed daily by clinical scoring and ankle size measurements until the endpoint (day 42). Ankle joints were assessed by a histopathologic score and microfocal computed tomography (micro-CT). Systemic effects of inflammation on bone metabolism were quantified by histomorphometry and micro-CT of the tibia.

Results: Acute Arthritis developed in both tg and WT mice and remained active over the period of 42 days, with a reduced, yet non-significant, severity in $\mathrm{tg}$ compared to WT mice. Histological indices of inflammation, cartilage damage and especially bone erosion, additionally assessed by micro-CT, tended to be overall reduced in tg mice, yet not reaching a level of significance. Bone volume and bone turnover did not differ between tg and WT arthritic mice.

Conclusions: The modulating effect of disrupted GC-signaling in osteoblasts in serum-induced autoimmune-arthritis prevails in a chronic inflammatory setting, leading to less severe local inflammation and bone destruction. This supports the important role of endogenous GCs for an intact bone metabolism in inflammatory bone disease.

References:

[1] Tu J, Zhang Y, Kim S, Wiebe E, Spies CM, Buttgereit F, et al. Transgenic Disruption of Glucocorticoid Signaling in Osteoblasts Attenuates Joint Inflammation in Collagen Antibody-Induced Arthritis. Am J Pathol. 2016;186(5):1293-301.

[2] Buttgereit F, Zhou H, Kalak R, Gaber T, Spies CM, Huscher D, et al. Transgenic disruption of glucocorticoid signaling in mature osteoblasts and osteocytes attenuates $\mathrm{K} / \mathrm{BxN}$ mouse serum-induced arthritis in vivo. Arthritis Rheum. 2009;60(7):1998-2007.

Disclosure of Interest: None declared

DOI: 10.1136/annrheumdis-2017-eular.5806
THURSDAY, 15 JUNE 2017

\section{Treat-to-target in axSpA: reality or utopy?}

\section{OP0188 DEFINING CLINICALLY IMPORTANT WORSENING BASED ON ASDAS-CRP FOR AXIAL SPONDYLOARTHRITIS: A DATA-BASED CONSENSUS BY THE ASSESSMENT IN SPONDYLOARTHRITIS INTERNATIONAL SOCIETY (ASAS)}

A. Moltó $^{1}$, L. Gossec ${ }^{2}$, R. Landewé ${ }^{3}$, D. van der Heijde ${ }^{4}$, M. Dougados ${ }^{1}$. ${ }^{1}$ Rheumatology B Department, Cochin Hospital; ${ }^{2}$ Pitié Salpêtrière Hospital, Department of rheumatology, Paris, France; ${ }^{3}$ Department of Clinical Immunology \& Rheumatology, Amsterdam Rheumatology Center, Amsterdam and Zuyderland Medical Center, Heerlen; ${ }^{4}$ Department of Rheumatology, Leiden University Medical Centre, Leiden, Netherlands

Background: Disease flares are increasingly used as outcomes in axial spondyloarthritis (axSpA) trials or observational studies.

Objectives: The objective of this initiative was to define a cutoff for the ASDAS score that best defines the concept of "worsening in axSpA", to be used in the context of clinical trials and longitudinal observational studies.

Methods: Various steps were followed between 2014 and 2017. (a) Initial expert opinion within the steering group to define the scope of the project; (b) systematic literature review to collect cutoffs used for worsening in published studies; (c) vignette-exercise among ASAS members: a theoretical 'paper' patient-vignette, in whom an initial and a final value of an outcome was provided, was judged by the physicians on whether or not the patient had worsened (defining phyworsening) (ref); (d) real-life multicenter international study: data necessary to calculate different outcomes were collected from real patients at 2 consecutive visits (spaced 7 days to 6 months): external standard was defined as a patient's report that he/she had worsened and he/she felt there was a need for treatment intensification. (e) Testing of different changes in the outcomes against both external standards for worsening (phy-worsening and pt-worsening) followed by a consensus and voting procedure among ASAS members in January 2017.

Results: (a) There was consensus about worsening being an absolute change between 2 time-points (without defining time between the 2 time-points) and about exploring cutoffs for 3 outcomes: ASDAS-CRP, BASDAI and pain. (b) The literature review had yielded 27 different cutoffs in 38 studies indicating important heterogeneity (c) The vignette-exercise yielded 12 preliminary definitions for worsening to be tested (as previously reported). (d) In the prospective study the sensitivity and specificity of each cutoff was tested against pt-worsening and judged by the ASAS-community. (e) No consensus was reached for a BASDAI-based definition due to limited performance of all cut-offs, and it was decided to not define a value for a pain-based definition for worsening. Based on aggregated data (Table), a consensus was reached among the ASAS-members to define worsening as a deterioration in ASDAS of at least 0.9 points. While this cutoff led to only moderate sensitivity when tested against pt-worsening, the overall balance of sensitivity and specificity as well as the overall face validity of this cut-off value for ASDAS was deemed most acceptable.

\begin{tabular}{|c|c|c|c|c|}
\hline \multirow[t]{2}{*}{$\begin{array}{l}\text { Cutoff values } \\
\text { for change in } \\
\text { ASDAS }\end{array}$} & \multicolumn{2}{|c|}{$\begin{array}{l}\text { Vignette exercise study } \\
(\mathrm{N}=1150 \text { physician judgments) } \\
\text { against the external standard } \\
\text { 'phy-worsening' (worsening in } \\
\mathrm{N}=591 \text { ) }\end{array}$} & \multicolumn{2}{|c|}{$\begin{array}{l}\text { Prospective real-life study } \\
\text { ( } N=1169 \text { patients), against the } \\
\text { external standard 'pt-worsening' } \\
\text { (worsening in } N=127 \text { ) }\end{array}$} \\
\hline & Sensitivity (\%) & Specificity (\%) & Sensitivity (\%) & Specificity (\%) \\
\hline$>=0.6$ & 97 & 65 & 55 & 91 \\
\hline$>=0.9$ & 85 & 87 & 38 & 96 \\
\hline$>=1.1$ & 60 & 94 & 33 & 98 \\
\hline
\end{tabular}

Conclusions: This data-driven ASAS consensus process has allowed to propose an ASDAS-based cutoff value defining worsening in axSpA. As has been observed in other settings, the change defining worsening (at least 0.9 ) is smaller than the change defining improvement which is 1.2 for ASDAS. This definition should now be applied in trials.

References:

[1] Gossec L, et al. Preliminary definitions of 'flare' in axial spondyloarthritis, based on pain, BASDAI and ASDAS-CRP: an ASAS initiative. Ann Rheum Dis. 2016;75:991-6.

Acknowledgements: This study was supported by ASAS.

Disclosure of Interest: None declared

DOI: 10.1136/annrheumdis-2017-eular.4786 